\section{A Restoration Method for Impulsive Functions}

\section{ROBERT W. CLAYTON AND TADEUSZ J. ULRYCH}

\begin{abstract}
A method is presented for enhancing the resolution of impulsive functions which have been degraded by a known convolutional disturbance and by the addition of white noise. An autoregressive model is employed to represent the spectrum of the ideally resolved impulsive function. The method is flexible in that it allows constraints to be incorporated into the resolution scheme. Two quite diverse examples are presented as illustration.
\end{abstract}

\section{INTRODUCTION}

In this paper, we present a method for increasing the resolution of functions which have as their ideal, "perfectly resolved" form, a sum of phase-shifted Dirac impulses. The degradation in resolution is assumed to be due to a convolutional disturbing function and the addition of white noise.

For the purposes of this discussion, we will assume that the disturbance function is known a priori. Generally this function has to be estimated from the data themselves or from an understanding of the physical processes that govern the observations.

With the disturbance known a priori, the essential problem reduces to estimating the parameter sets $\left\{A_{j}\right\}$ and $\left\{Q_{j}\right\}$ of the following model for the impulsive function:

$$
h_{q}=\sum_{j}^{n} A_{j} \delta\left(q-Q_{j}\right)
$$

where $q$ is the discrete index of the sampled series, $A_{j}$ is the amplitude of the $j$ th impulse, and $Q_{j}$ is the offset in $q$ of the $j$ th impulse. The model for $h_{q}$ is assumed to be related to the observations by

$$
x_{q}=s_{q} \otimes h_{q}+n_{q},
$$

where $x_{q}$ is the observed function, $s_{q}$ is the disturbance function, and $n_{q}$ is white noise.

Conventionally, there are two approaches to the determination of $\left\{A_{j}\right\}$. The first of these is the nonparametric approach which includes Wiener deconvolution, predictive deconvolution, and spectral division. These methods are nonparametric in the sense that they do not use the model of the impulse function. They simply attempt to transform $x_{q}$ into an estimate of $h_{q}$ by removing, as much as possible, the effects of the disturbance function. For example, with spectral division, $h_{q}$ is estimated by

$$
h_{q}=F^{-1}\left(X_{r} / S_{r}\right)
$$

where the capitals denote the Fourier transform pairs of the quantities in (2), $r$ is the discrete index of the transformed domain, and $F^{-1}(\cdot)$ is the inverse Fourier transform. Due to noise enhancement problems, this estimator has to be limited to regions where $S_{r}$ has significant spectral energy, and usually the estimator is set to zero outside this band. The other nonparametric methods have similar restrictions. If the deconvolution is limited so that $r \in\left[r_{a}, r_{b}\right]$, where $r_{a}$ and $r_{b}$ are the lower and upper bounds of significant spectral energy of $S_{r}$, then the resolution that is achieveable is proportional to $1 /\left|r_{a}-r_{b}\right|$. If $\left|r_{a}-r_{b}\right|$ is small with respect to the total bandwidth, then the resolution will be poor, and consequently the ability to pick the parameters $\left\{A_{j}\right\}$ and $\left\{Q_{j}\right\}$ is limited.

Manuscript received April 26, 1976; revised August 5, 1976.

The authors are with the Department of Geophysics and Astronomy, the University of British Columbia, Vancouver, BC, Canada V6T 1 W5.
The second approach is the parametric approach, which attempts to fit the impulse model directly to the observations under some error-of-fit criterion. For example, minimizing the least squared error between the observations and the model formed by the combination of (1) and (2) leads to the following set of equations:

$$
\begin{gathered}
\sum_{q}\left[s\left(q-Q_{k}\right)\right] e_{q}=0, \quad k=1 \cdots n \\
\sum_{q}\left[\frac{\partial s\left(q-Q_{k}\right)}{\partial Q_{k}}\right] e_{q}=0, \quad k=1 \cdots n,
\end{gathered}
$$

where $e_{q}$ is the local error, defined to be

$$
e_{q}=x_{q}-\sum_{j}^{n} A_{j} s\left(q-Q_{j}\right)
$$

The second of these equations is generally nonlinear, and also has the unfortunate property that the most reliable observations are not the ones which are most heavily weighted. This is seen from the fact that if the local signal-to-noise ratio is defined as $\left|x_{q}\right|^{2} / \sigma_{n}{ }^{2}$, where $\sigma_{n}{ }^{2}$ is the noise variance, then the most reliable observations occur where $\left|x_{q}\right|$ is large. The second equation weights the local error (and hence the observations) most heavily at the points of maximum slope, which is not necessarily at the points of maximum signal-to-noise ratio.

These two problems make it difficult in practice to determine both the $\left\{A_{j}\right\}$ and $\left\{Q_{j}\right\}$ parameters. If the $\left\{Q_{j}\right\}$ can be determined by alternative means, then only (4) is necessary to determine the $\left\{A_{j}\right\}$. This equation is linear in $\left\{A_{j}\right\}$, and also weights the most accurate observations most heavily. Another significant problem with the parametric approach is the requirement of determining the number of parameters for the model.

The method we propose attempts to combine features of both the nonparametric and parametric approaches. The main emphasis of the method will be to find the $\left\{Q_{j}\right\}$ parameters. This method is outlined in the next section. In the last section, we present two examples which illustrate the method.

\section{METHOD}

The ideally resolved output is modeled as an impulsive function. In order to include the case of phase-shifted impulses, we will use the analytic form of $x_{q}$, which is defined to oe [1]

$$
x_{q}=x_{q}+i H\left(x_{q}\right)
$$

where $H(\cdot)$ is the Hilbert transform. If $s_{q}$ and $n_{q}$ are real functions, then $\hat{x}_{q}$ is related to $\hat{h}_{q}$ in a form analogous to (2) as

$$
\hat{x}_{q}=s_{q} \otimes \hat{h}_{q}+\hat{n}_{q} \text {. }
$$

Since a phase-shifted impulse is expressible as a rotation of the analytic form of the impulse, $\hat{h}_{q}$ has the following form

$$
\hat{h}_{q}=\sum_{j}^{n} A_{j} e^{i \epsilon_{j}}\left[\delta\left(q-Q_{j}\right)+i H\left(\delta\left(q-Q_{j}\right)\right)\right]
$$

where $\left\{\epsilon_{j}\right\}$ are the phase-shift angles. This expression has a simpler form when it is Fourier-transformed, namely,

$$
\hat{H}_{r}=\sum_{j}^{n} A_{j} e^{i\left(r Q_{j}+\epsilon_{j}\right)}(1+\operatorname{sgn}(r)),
$$

where $\operatorname{sgn}(r)=1$, when $r \geq 0$, and $\operatorname{sgn}(r)=-1$, otherwise.

The first step in the enhancement process is to remove, as much as possible, the effects of the disturbance function. To accomplish this we employ the spectral division of (3). The deconvolution is restricted to $r \in\left[r_{a}, r_{b}\right]$, the band of significant spectral energy of the disturbance function.

The next step is to use the model of the impulsive function to predict the behavior of the function for $r \in\left[r_{a}, r_{b}\right]$. An examination of (10) indicates that the spectral form of the model of the impulsive function is a sum of complex sinusoids. This means 
that any subset of $\hat{H}_{r}$, say $\left\{\hat{H}_{r} \mid r \in\left[r_{a}, r_{b}\right]\right\}$, is characteristic of the whole set in the sense that the second-order statistics are the same. This is the condition that is necessary to cast $\hat{H}_{r}$ as an autoregressive (AR) sequence. The suitability of applying AR models to complex sinusoids has been discussed by Ulrych and Clayton [2].

The general form of the AR model is

$$
\hat{H}_{r}=\sum_{k=1}^{m} \alpha_{k} \hat{H}_{r-k}+I_{r}
$$

where $\left\{\alpha_{k}\right\}$ are the AR coefficients and $I_{r}$ is the innovation. The subset $\left\{\hat{H}_{r} \mid r \in\left[r_{a}, r_{b}\right]\right\}$, which is estimated from $\hat{X}_{r}$ by spectral division with $S_{r}$, can be used as a data base to fit the coefficients of (11). Several methods of fitting the AR model are possible, and the suitability of the various techniques is discussed in Ulrych and Clayton [2]. For the examples presented in this paper, the Burg algorithm [3] was used.

Once the $\left\{\alpha_{k}\right\}$ have been determined, the AR model may be applied in a unit-step recursive fashion to predict the unknown spectral regions of $\hat{H}_{r}$. Prediction by this method preserves the phase-shift angle information of (10), and, consequently, when $\hat{H}_{r}$ is transformed back into time $q$-domain, its real part correctly reflects the phase shifts of the impulses. The energy of the predicted $\hat{H}_{r}$ decays because the innovation is not included in the prediction.

One important parameter that must be chosen in the scheme is the order of the AR model that is to be used. The choice of this parameter is much less critical than the choice of the number of impulses to be included in the totally parametric approach. Generally, using an AR order that is too low produces an underresolved estimate of $h_{q}$, while overestimating the order introduces spurious noise components. The final prediction error (FPE) criterion and the automatic information criterion (AIC) have been introduced by Akaike [4], [5], to provide a method for choosing the optimum AR order. However, neither of these criteria are directly applicable to purely harmonic sequences. We have found that, for sinusoids, both criteria underestimate the order that provides optimum resolution. The FPE, AIC, and their relation to sinusoidal processes are discussed in Ulrych and Clayton [2].

\section{SPECIAL CASES}

If the impulses of the impulsive function are not phase-shifted, or the phase-shifted angle information is not needed, then the procedure outlined above may be improved. Specifically, $h_{q}$ can be estimated by simply computing the amplitude spectrum of the AR model itself, rather than using the model to predict the unknown spectral regions of $\hat{H}_{r}$. The amplitude spectrum of the AR model is

$$
h_{q}=\frac{P^{1 / 2}}{\left|1-\sum_{k=1}^{m} \alpha_{k} e^{i 2 \pi k q}\right|}
$$

where $P$ is the prediction error power. This estimator will generally be more resolved than the method outlined above, because it is independent of the data that does not conform to the model.

If $x_{q}$ can be considered a power spectrum, that is, $h_{q}$ contains only positive impulses and $s_{q}$ is a strictly positive function, a further modification can be made since then the Fourier transform of $x_{q}$ will be an autocovariance function. The importance of including the positive constraint in the restoration scheme for functions that have this property has been shown by Frieden [6], [7]. After the effects of the disturbance function have been removed from $x_{r}$, it can be used directly in the Yule-Walker equations [8] as an estimate of the autocovariance. The impulsive function is estimated by computing the AR power spectrum which is the square of the AR amplitude spectrum given in (12)

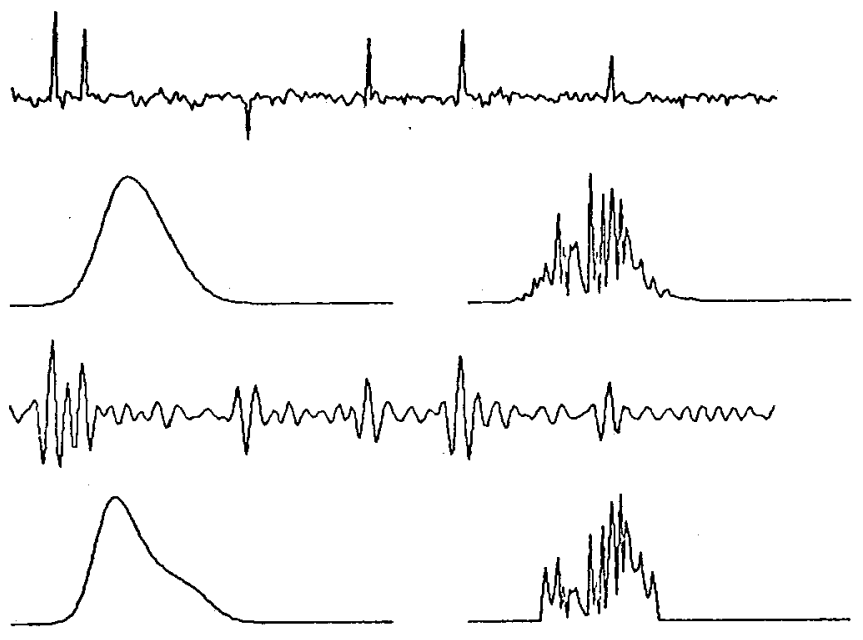

Fig. 1. Details of first example. Shown are ideal impulse sequence, amplitude spectrum of attenuation function, resultant synthetic trace, and its amplitude spectrum. Last two plots show estimated attenuation function spectrum and trace amplitude spectrum after spectral division. All spectra extend from zero to $\mathrm{Ny}$ quist frequency.
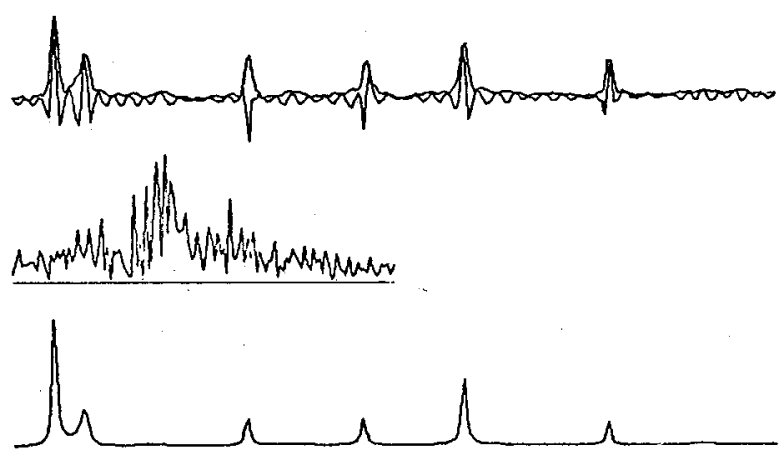

Fig. 2. Enhancement procedure is applied to synthetic trace in Fig. 1. First plot shows enhanced impulse sequence with its superimposed envelope. Second plot is its amplitude spectrum showing spectral extension. Third plot is amplitude spectrum of AR model used in prediction.

\section{EXAMPLES}

We present two synthetic examples to illustrate the method. The first of these examples is typical of the processes used in Vibroseis reflection seismology [9] and in Chirp radars [10]. A swept-frequency FM signal is used as a source to excite the impulse response of the medium. The source is then cross-correlated with the recorded signal to produce a process which can be modeled as the impulse response of the medium convolved with the autocorrelation of the FM sweep. Ideally, the amplitude spectrum of the autocorrelation of the FM sweep is flat within the sweep band. To make the example somewhat more realistic, the attenuation and dispersion properties of the medium are included by modifying the spectrum of the signal with a Gaussian function. The ideal impulse response, with some added white noise, and the amplitude spectrum of the attenuation function are shown in Fig. 1. The synthetic trace and its amplitude spectrum are also shown in this figure.

The attenuation function was estimated by assuming that it is a low frequency phenomenon compared with the impulse response. Consequently, it can be estimated by low-passing the spectrum of the synthetic trace [11], [12]. The result of removing the attenuation function by spectral division is shown in the latter part of Fig. 1.

The spectrum was extended by prediction with an AR model as described in the method above. An AR order of 12 was used, 


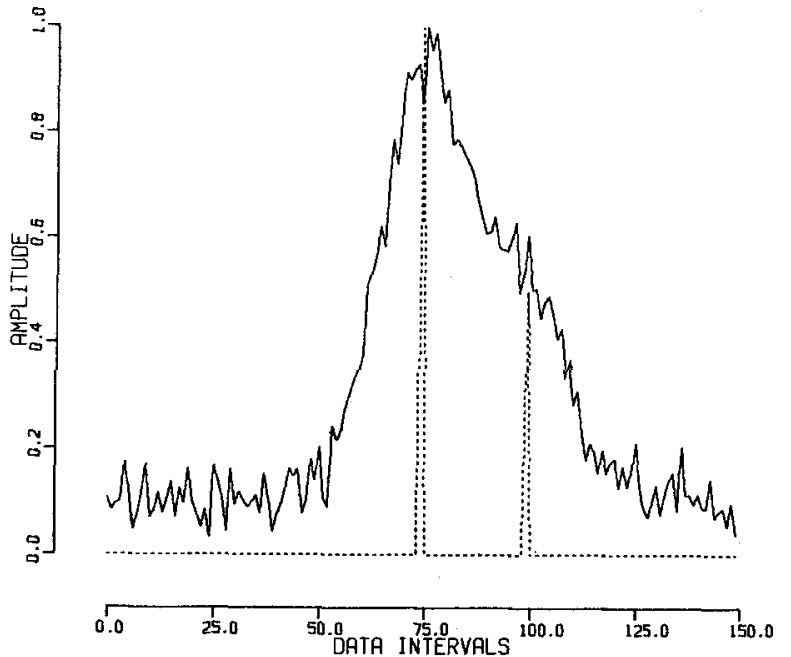

Fig. 3. Synthetic astronomical line spectrum. Ideally resolved impulses are shown as dotted lines.

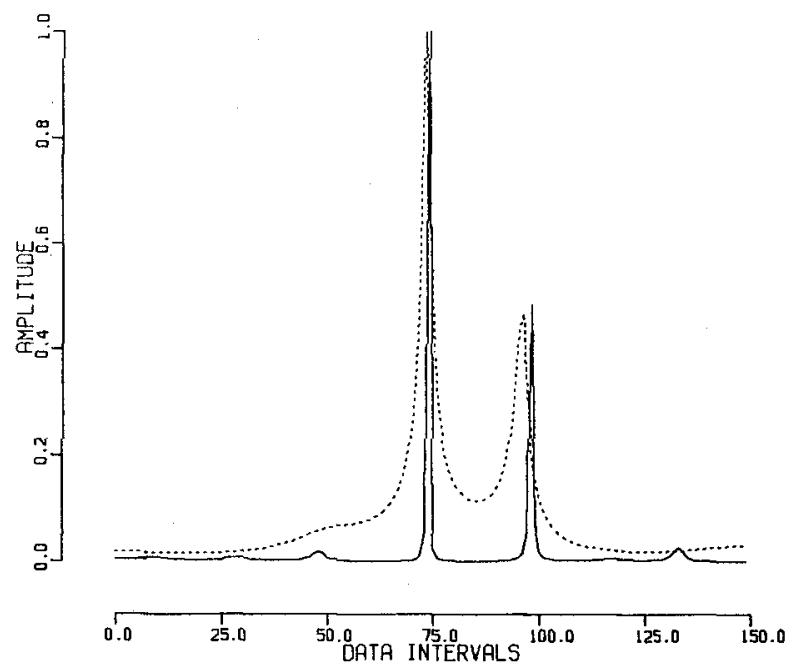

Fig. 4. Enhanced lihe spectrum. Dotted line is amplitude spectrum of AR model Solid line is enhanced impulses when input signal is assumed to be power spectrum.

and the results are shown in Fig. 2. In this figure, the enhanced impulse series is shown with its superimposed envelope, which is defined as the modulus of the analytic signal. The predicted spectrum and the amplitude spectrum of the AR model are also shown.

The second example is that of a synthetic astronomical line spectrum. Doppler motions within the stars cause the theoretical spectral lines to be broadened in a way which can be modeled by a Gaussian function [13]. The example was constructed by the convolution of a Gaussian function with two Dirac impulses and the addition of white noise. The mean of the process was adjusted so that the signal is strictly positive. The synthetic signal and the generating impulses are shown in Fig. 3. The spectral division was done with a Gaussian function whose half-width was estimated from the data [14].

Both methods outlined in the special cases section were applied to the example and the results are shown in Fig. 4 . The method which assumes the observed signal is actually a power spectrum produced the greatest resolution. As a comparison with conventional nonparametric methods, the signal was deconvolved, but not fitted to an AR model. The result, as shown in Fig. 5, is a marked decrease in resolution and the introduction of negative sidelobes. The restoration of real astronomical spectral data using

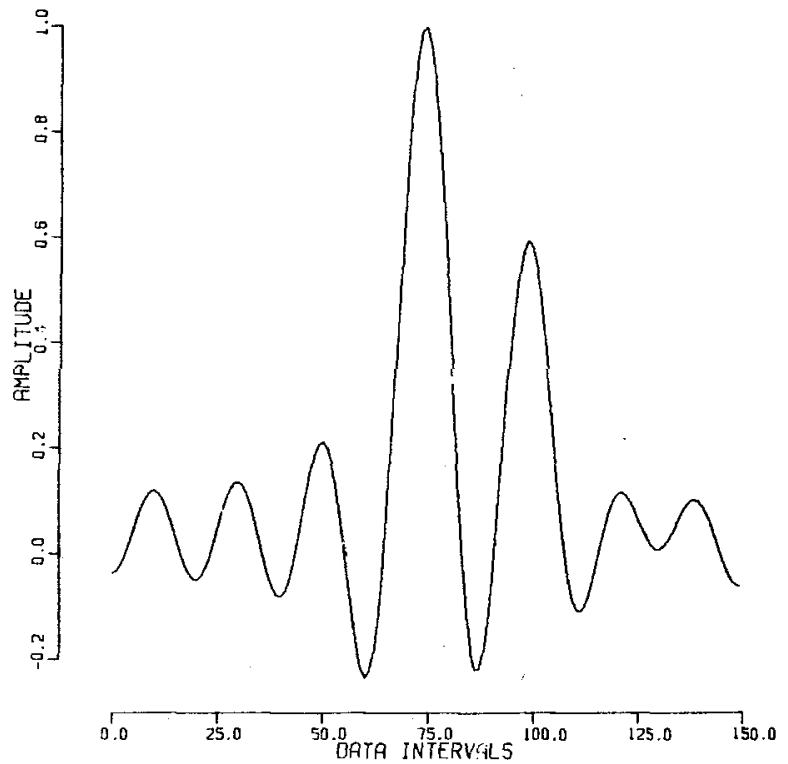

Fig. 5. Figure indicates resolution that is achieveable when spectral division is applied without AR modeling.

the described technique shows a similar resolution enhancement to that in the example shown here [15]. The reliability of the technique has been demonstrated on synthetic as well as real data.

\section{Conclusions}

This paper describes a restoration method which is based on modeling the spectrum of the ideally resolved output as an AR process. This method is readily adaptable to the data on hand. Specifically, a positive constraint is incorporated into the scheme where such a constraint applies or is desirable. The two examples presented demonstrate the usefulness of the technique in quite diverse applications.

\section{REFERENCES}

[1] R. Bracewell, The Fourier Transform and its Application. New York: McGraw-Hill, pp. 267-272, 1965.

[2] T. J. Ulrych and R. W. Clayton, "Time series modelling and maximum entropy," in press, Phys. Earth Planet. Int., 1976.

[3] J. P. Burg, "Maximum entropy spectral analysis," Ph.D. dissertation, Stanford University, Stanford, CA, 1975.

[4] H. Akaike, "Statistical predictor identification," Ann. Inst. Statist. Math., vol 22, pp. 203-217, 1970 .

[5] _- "A new look at the statistical model identification," IEEE Trans. Automat. Contr., vol. AC-19, pp. 716-723, 1974

[6] B. R. Frieden, "Restoring with maximum likelihood and maximum entropy," J. Opt. Soc. Am., vol. 62, pp. 511-518, 1972.

[7] - - in Topics in Applied Physics, (Ed. T. S. Huang). New York: Springer-Verlag, vol. 6, pp. 219-245, 1975

[8] T. J. Ulrych and T. N. Bishop, "Maximum entropy spectral analysis and autoregressive decomposition," Rev. Geophys., vol. 13, pp. 183-200, 1975.

[9] D. Ristow and D. Jurczyk, "Vibroseis deconvolution," Geophys. Prosp., vol. 23, pp. 363-379, 1975.

[10] J. R. Klauder, A. C. Price, S. Darlington, and W. J. Albersheim, "The theory and design of Chirp radars," The Bell System Technical Journal, vol. 39, pp. $745-808,1960$

[11] A. V. Oppenheim, R. W. Schafer, and T. G. Stockham, "Nonlinear filtering of multiplied and convolved signals," Proc. IEEE, vol. 65, pp. 1264-1291, 1968.

[12] T. J. Ulrych, "Application of homomorphic deconvolution to seismology," Geophysics, vol. 36, pp. 650-660, 1971.

[13] K. R. Lang, Astronomical Formulae. New York: Springer-Verlag, pp. 204-206, 1974.

[14] T. J. Ulrych, J. R. Auman, J. A. Eilek, and G. A. H. Walker, "Resolution enhancement of astronomical spectra," Astron. and astrophys., vol. 21, pp. 125-130, 1972.

[15] A. Allison, "Spectral variations of 12 lacertae," M.Sc. thesis, University of British Columbia, Vancouver, BC, Canada 1976. 